\title{
Effect of Size and Shape on Static Refractive Index, Dielectric constant and Band gap of Nano solids
}

\author{
G.R. Patel ${ }^{1 *}$ and T.C. Pandya ${ }^{2}$ \\ ${ }^{1 *}$ Physics Department, Government Science College, Gandhinagar, Gujarat, India \\ ${ }^{2}$ Physics Department, St. Xavier's College, Navrangpura, Ahmedabad, Gujarat, India \\ *Corresponding Author: grpalp94@gmail.com, Tel.: +91-94278-38047
}

Available online at:www.isroset.org

Received 26/Dec/2017, Revised 10/Jan/2018, Accepted 21/Feb/2018, Online 28/Feb/2018

\begin{abstract}
In the present study, a simple theoretical model has been proposed for computation of optical properties such as static refractive index, static dielectric constant and band gap for nanosolids having different nanostructures of different shape based on size dependent atomic cohesive energy. We have computed size dependent static dielectric constant for silicon nanofilm and deviation in band gap for Si nanowire and nanofilm for different size. We have also computed size dependent bandgap of Ge nanowire having square cross section. We have also found that band gap increases as particle size decreases. Our results are compared with the available experimental and simulated data. A close agreement of the computed results with available experimental and simulated data validates the present theoretical model. The present relationships of Static Refractive Index, Dielectric constant and Band gap of Nano solids are potentially applicable for those materials whose experimental data are not available.
\end{abstract}

Keywords- Band-gap, refractive index, dielectric constant, size and shape dependent, $\mathrm{Si}, \mathrm{Ge}$

\section{INTRODUCTION}

To describe the optical and electronic properties of a semiconductor's refractive index, dielectric constant and energy gap are fundamental physical aspects. For semiconductors like Silicon $(\mathrm{Si})$ and Germanium $(\mathrm{Ge})$ the parameters including the melting temperature $T_{m}$, static refractive index $\eta$, static dielectric constant $\varepsilon$ and band gap $E_{\mathrm{g}}$ are primarily important to develop devices [1]. If the size of the material is reduced to the nanoscale range, the parameters of the material becomes tunable due to size and shape dependence. Furthermore, the trends of the change of parameters with size and shape are different from each another. Reducing the diameter (D) of nanoparticles, the most common observations found for Si nanocrystals are (1) static refractive index $\eta(D)$ and static dielectric constant $\varepsilon(D)$ reduces [2]. Reduction in static dielectric constant $\varepsilon(D)$ causes an increase in Coulomb interaction energy between electrons, holes and ionized impurities; this increment, can considerably modify the optical absorption and transport phenomenon of nanoscale devices [2]. (2) Reduction in diameter (D) causes an increase in Bandgap $E_{\mathrm{g}}(D)$ which is an important parameter when we are dealing with the semiconductors [2]. Since the physical and chemical properties of the nanomaterials are depending on the bandgap of nanosolids, the change in bandgap alters these properties considerably. To understand these unusual properties of nanomaterials, a lot of theoretical models have been proposed from various perspectives. For the study of size induced melting temperature $T_{m}$ depression, the liquid drop model, W. Qi model, latent heat model and bond energy model have been proposed [3-7]. The size-induced $E_{\mathrm{g}}$ expansion can be explained in terms of the quantum confinement premise [8]. Suppression of static dielectric constant is due to the breaking of surface polarizable bonds [9]. Guisbiers et al. [10] have expressed the melting temperature and bandgap of nanomaterials as function of its diameter and the shape parameter.

Zhang [11] also proposed a core-surface (CS) model to explain the size effects on the Young's modulus and dielectric constant, where the nano wire is modeled as a composite beam consisting of the core section of the bulk material and the surface layer. Generally, the size effect is originating from the surface-to-volume ratio effect and the statistics followed by the particles involved in the material property [12]. However, the above-mentioned different theoretical interpretations indicate that there are different rules governing the size dependences of these physical parameters.

In the literature, we could not find any model involving shape factor of given nanostructure for computing size and shape dependent static refractive index, static dielectric constant and band gap. In computation of these parameters shape factor plays very important role, because increment in 
shape factor enhances surface to volume ratio which alters the above-mentioned properties.

In the present paper, we have reported a simple theoretical model for the computation of size and shape dependence of the static refractive index, static dielectric constant and bandgap of nanosolids. We have also analyzed the effect of shape factor for a given shape of a nanosolid. Our computed results agree well with the available experimental and simulated results which gives the validity to our work.

The formulation for computation of size and shape dependence of the static refractive index, static dielectric constant and bandgap of nanosolids with taking care of shape factor for given shape of nanosolids is described in the section II of this paper. Comparison of our computed results with available experimental and simulated results are given in section III. Conclusion drawn from our computed results are mentioned in section IV.

\section{METHOD OF ANALYSIS}

Static refractive index for a bulk material is given by [13]

$$
\eta-1=\frac{N q_{e}{ }^{2}}{2 \varepsilon_{0} m \omega_{0}{ }^{2}}
$$

Where $\mathrm{N}$ is number of atoms per unit volume and $\omega_{o}$ is the natural angular frequency of electrons in atoms of the bulk material, $\mathrm{m}$ is the mass of the electron and $\mathrm{q}_{\mathrm{e}}$ is the charge of the electron, it is supposed that electrons behave as if they are held by a spring; so we will consider that the electrons behave like little oscillators with a resonant frequency $\omega_{o}$.

Parameters $\mathrm{N}$ and $\omega_{o}$ are size dependent parameters. At the nano scale range the surface to volume ratio becomes very large, so numbers of atoms at the surface are very large in comparison to volume. Hence, for nanomaterial, the number of atoms per unit volume decreases. At the nano scale range electron orbits contracts that causes the increment in the Columbian force which enhances restoring force. This increment in restoring force causes the increment in the natural angular frequency of electron oscillation.

No. of atoms inside the volume of nanosolid is given by [5]

$$
\frac{n_{D}}{n}=\left(1-\frac{N_{s}}{2 n}\right)
$$

Where $n_{D}$ and $n$ are the number of atoms inside the volume of nanomaterials and bulk material respectively and $N_{s}$ is the number of surface atoms on the nanomaterial.

Here the ration $\mathrm{Ns} / \mathrm{n}$ is $4 \mathrm{~d} / \mathrm{D}$ for spherical nanosolids, $(8 / 3) d / D$ for nanowire and $(4 / 3) d / h$ for nanofilm.
Where $\mathrm{d}$ is the diameter of the atom, $\mathrm{D}$ is the diameter of the nanoparticle, nanowire and $\mathrm{h}$ is the thickness of the nanofilm.

It is found that natural oscillation angular frequency $\omega_{o}$ of the electrons and vibrational frequency of phonons in the nanomaterials are same [14]. Hence size dependent natural oscillation angular frequency $\omega_{o}$ of the electrons in the atoms of the nanomaterials is given by [15]

$$
\frac{\omega_{0(D)}}{\omega_{0}}=\frac{h}{h_{(D)}}\left[\frac{E_{(D)}}{E}\right]^{\frac{1}{2}}
$$

Where,

$$
\frac{h_{(D)}}{h}=\frac{E_{(D)}}{E}=\left(1-\frac{\beta \alpha}{D}\right)
$$

Where $\omega_{o(D)}$ is the size dependent vibrational frequency of the electron in atoms of the nanomaterial and $\omega_{o}$, the vibrational frequency of the electrons in atom of the corresponding bulk material, $h$ and $h_{(D)}$ are bond length of bulk and nanomaterials respectively, $E_{(D)}$ and $E$ are size dependent and bulk cohesive energy. Here $\alpha$ is the dimensionless shape factor, which is defined by the ratio of the area of the nanoparticle in any shape whose volume is the same as the spherical nano particle to the area of the spherical nanoparticle [16]. It is 1 for spherical, 1.49 for regular tetrahedral and 1.24 for regular hexahedral nano particle. For a nanowire, it is defined by the ratio of the surface area of the nanowire with arbitrary cross sectional shape whose volume is the same as the cylindrical nanowire to the surface area of the cylindrical nanowire [17]. It is 1.13 for nanowire with rectangular cross-section.

In the present work, for computing size dependent cohesive energy and melting temperature, we have used our welldeveloped size and shape dependent cohesive energy or melting temperature model for elemental nanocrystals [18] which has been deduced as

$$
\frac{E_{m n(D, \alpha)}}{E_{m b}}=\frac{T_{m n(D, \alpha)}}{T_{m b}}=1-\frac{\beta \alpha}{D}
$$

$\mathrm{T}_{\mathrm{mn}(\mathrm{D}, \alpha)}$ is the size and shape dependent melting temperature of the nanoparticle, $\mathrm{T}_{\mathrm{mb}}$ is the melting temperature of the corresponding bulk material, $\mathrm{D}$ is the diameter of the nanoparticle and $\beta$ is material dependent constant [18]. Equation (5) can be written for size and shape dependent melting for pancake-shaped nanoparticles. 


$$
\frac{E_{m n(D, \alpha)}}{E_{m b}}=\frac{T_{m n(D, \alpha)}}{T_{m b}}=1-\frac{\beta \alpha A}{6}
$$

Here $A$ is the surface to volume ratio and for a spherical nanoparticle, it is given by

$$
A=\frac{3}{R}=\frac{6}{D}
$$

for a particle with cylindrical geometry of height $h$ and diameter $D$, the surface to volume ratio (A) is:

$$
A=\frac{4}{D}+\frac{2}{h}
$$

For thin wires $h \gg D$ so $A=4 / D$

For thin films $h<<D$ so $A=2 / h$

Using Eqs. (2), (3), (4), (5) and (6) equation (1) takes the empirical form for nano materials

$$
\frac{\left(\eta_{D}-1\right)}{(\eta-1)}=\left(1-\frac{\beta \alpha}{D}\right)\left(1-\frac{N_{S}}{2 n}\right)
$$

Empirical form of Size and shape dependent refractive index for a spherical nanoparticle

$$
\eta_{D, \alpha}=1+(\eta-1)\left(1-\frac{\beta \alpha}{D}\right)\left(1-\frac{N_{S}}{2 n}\right)
$$

For pancake-shaped nanoparticles

$$
\eta_{D, \alpha}=1+(\eta-1)\left(1-\frac{\beta \alpha A}{6}\right)\left(1-\frac{N_{s}}{2 n}\right)
$$

Size and shape dependent dielectric constant [19]

$$
\varepsilon\left({ }_{D, \alpha}\right)=\left(\eta_{D, \alpha}\right)^{2}
$$

To formulate the size and shape dependent band gap of Semiconductors, a well-known Arrhenius expression [1] for the size and temperature dependent electrical conductivity $\mu$ $(D, T)$ is introduced:

$$
\mu_{D, T}=\mu_{0(D)} \exp \left[\frac{-E_{a(D)}}{R T}\right]
$$

Where $\mu_{0}$ denotes a pre-exponential constant. $\mathrm{R}=\mathrm{N}_{\mathrm{A}} \mathrm{k}_{\mathrm{b}}$ where $\mathrm{N}_{\mathrm{A}}$ is Avogadro number and $\mathrm{k}_{\mathrm{b}}$ is Boltzmann constant. The activation energy for electrical migration is $E_{a}=E_{c}-E_{f}$ with $E_{c}$ and $E_{f}$ being the conductionband energy and the Fermi energy. In many semiconductors, $E_{f}$ is near mid-gap and thus $E_{a} \approx \frac{E_{g}}{2}$, where $E_{g}$ is the band gap energy [19]. If the change of $E_{g}$ is supposed to be proportional to the change of $E_{a}$, there is $\Delta E \mathrm{~g}(D) / E \mathrm{~g}_{\mathrm{b}}=$ $\left|\Delta E \mathrm{a}(D) / E \mathrm{a}_{\mathrm{b}}\right|$ where $\Delta$ denotes the change. Here $E \mathrm{~g}_{\mathrm{b}}$ and $E \mathrm{a}_{\mathrm{b}}$ are the corresponding band gap and activation energies of bulk materials.

If the electrical conductivity at the melting temperature is the same, independent of the melting temperature and therefore independent of the size, one can acquire the expression [1].

$$
\begin{gathered}
\frac{\Delta E_{g_{(D)}}}{E_{g b}}=\frac{\Delta E_{a(D)}}{E_{a b}} \\
\mu\left[D, T_{m}(D)\right]=\mu\left[b, T_{m b}\right] \\
\mu_{0(D)} \exp \left[\frac{-E_{a(D)}}{R T_{m(D)}}\right]=\mu_{0 b} \exp \left[\frac{-E_{a b}}{R T_{m b}}\right]
\end{gathered}
$$

Since the effect of the exponential term of $\exp \left[-E \mathrm{a}_{(\mathrm{D})} /(R T)\right]$ on $\mu_{(\mathrm{D}, \mathrm{T})}$ is much stronger than the effect of $\mu_{0(\mathrm{D})}$, as a first-order approximation, one can suppose that $\mu_{0(\mathrm{D})} \approx \mu_{0}(\mathrm{~b})$, where $\mu_{0}(\mathrm{~b})$ is the size independent preexponential constant.

Therefore, there is

$$
\begin{aligned}
\frac{E_{a(D)}}{E_{a b}} & =\frac{T_{m n(D)}}{T_{m b}} \\
\frac{\Delta E_{g_{(D, \alpha)}}}{E_{g b}} & =1-\frac{T_{m n(D, \alpha)}}{T_{m b}}
\end{aligned}
$$

Using Eq. (5) in Eq. (17) for spherical nanoparticles,

$$
\frac{\Delta E_{g_{(D, \alpha)}}}{E_{g b}}=\left[1-\left(1-\frac{\beta \alpha}{D}\right)\right]
$$

For pancake -shaped nanoparticles,

$$
\frac{\Delta E_{g_{(D, \alpha)}}}{E_{g b}}=\left[1-\left(1-\frac{\beta \alpha A}{6}\right)\right]
$$

For nanowire,

$$
\frac{\Delta E_{g_{(\alpha, D)}}}{E_{g b}}=\left[1-\left(1-\frac{2 \beta \alpha}{3 D}\right)\right]
$$


For nanofilm,

$$
\frac{\Delta E_{g_{(\alpha, D)}}}{E_{g}}=\left[1-\left(1-\frac{\beta \alpha}{3 h}\right)\right]
$$

\section{RESULT AND DISCUSSION}

Input parameters required for computation for silicon and germanium are compiled in table1.

Table 1

\begin{tabular}{|l|l|l|l|l|}
\hline & $\boldsymbol{\beta ( n m )}$ & $\begin{array}{l}\text { Band } \\
\text { gap(eV) }\end{array}$ & $\begin{array}{l}\text { Dielectric } \\
\text { constant }\end{array}$ & $\begin{array}{l}\text { Atomic } \\
\text { diameter(nm) }\end{array}$ \\
\hline $\mathrm{Si}$ & 0.8485 & 1.2 & 11.4 & 0.222 \\
\hline $\mathrm{Ge}$ & 1.06 & 0.67 & - & - \\
\hline
\end{tabular}

Results of static dielectric constant for Si film computed using Eq.11 are compared with experimental and simulated data in Fig.1. It predicts that below the film thickness of 5 $\mathrm{nm}$ size, static dielectric constant $\varepsilon(h)$ decreases rapidly. Our results are in good agreement with experimental and simulated data.

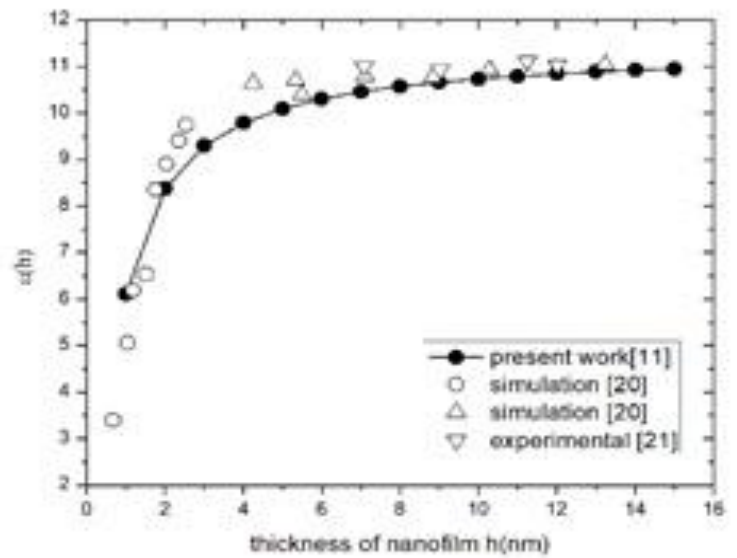

Fig.1: Size dependence of static dielectric constant for Si film.

Results of change in band gap $\Delta E g(D)$ for Si nanowire and nanoparticle computed using Eq.18 and Eq.19 are compared with the available experimental and simulated results shown in Fig. 2 and Fig. 3 respectively.

It is observed that change in bandgap is more rapid below the particle $3 \mathrm{~nm}$ size. Our results are in good agreement with experimental and simulated data.

Furthermore, deviation of $\Delta E \mathrm{~g}(D)$ for the nano wires versus $\mathrm{D}$ is weaker in comparison to nanoparticles. These differences should be attributed to the different surface to volume ratios $A=6 / D, 4 / D, 2 / h$ for nanoparticles, nanowires and nanofilms respectively.

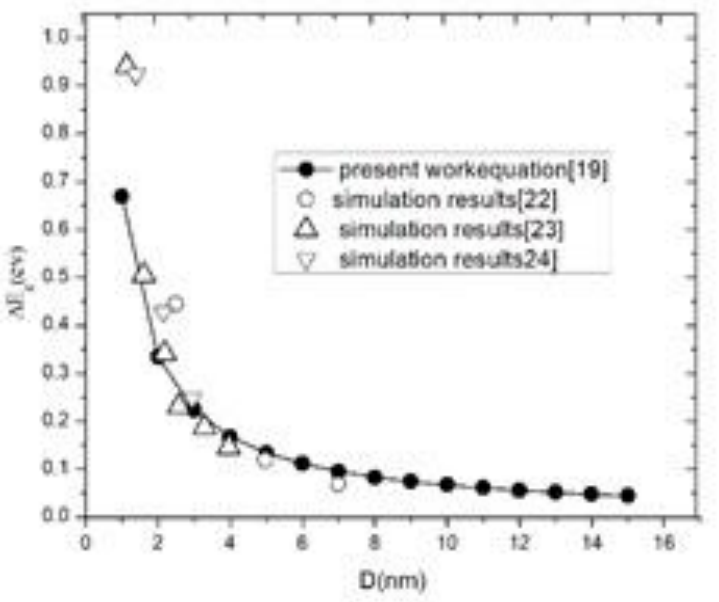

Fig.2: Deviation of size dependent band gap for Si nanowire.

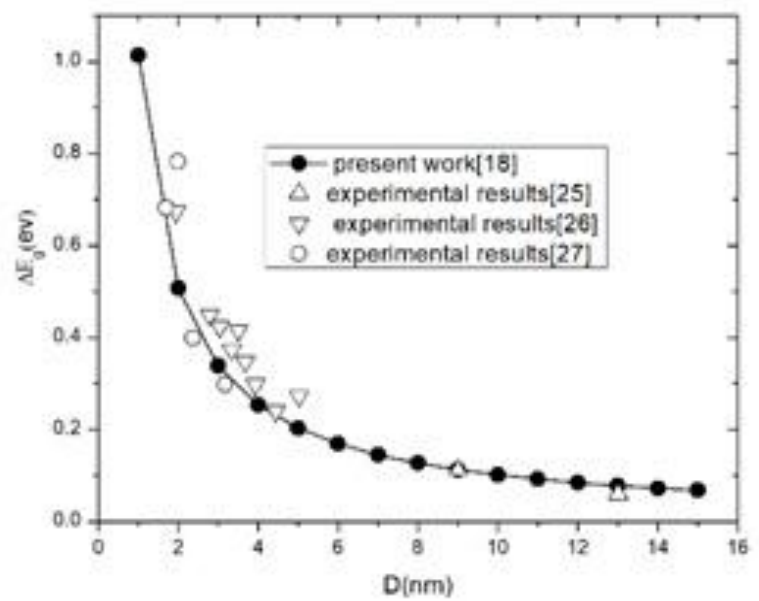

Fig.3: Deviation of size dependent band gap for Si nano particles.

Results of size dependent band gap $\mathrm{E}_{\mathrm{g}}(\mathrm{D})$ for Ge nanowire with square cross section computed using Eq.19 are compared with the available experimental and simulated results are reported in Fig.4.

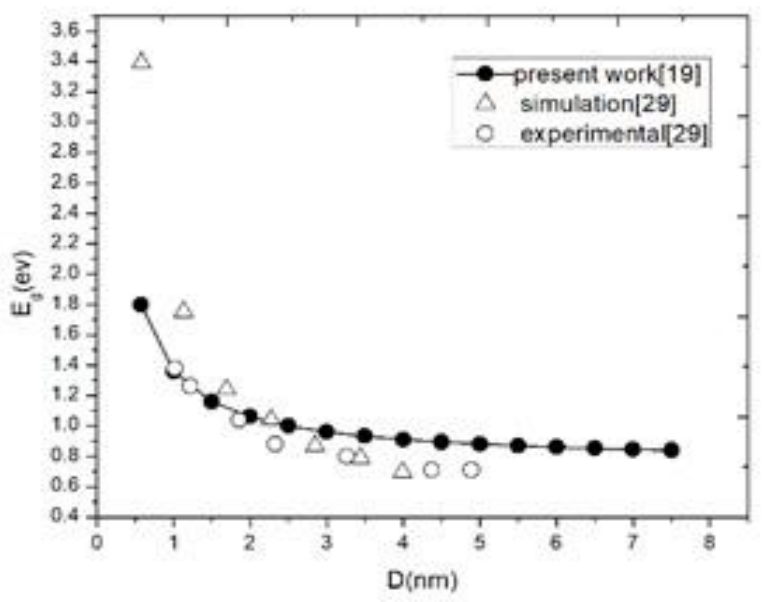

Fig. 4: Size dependent band gap for Ge nanowire. 
Deviation of band gap $\Delta E g(D, \alpha)$ versus shape factor for $\mathrm{Si}$ nanoparticle computed using Eq.18 are shown in Fig.5.

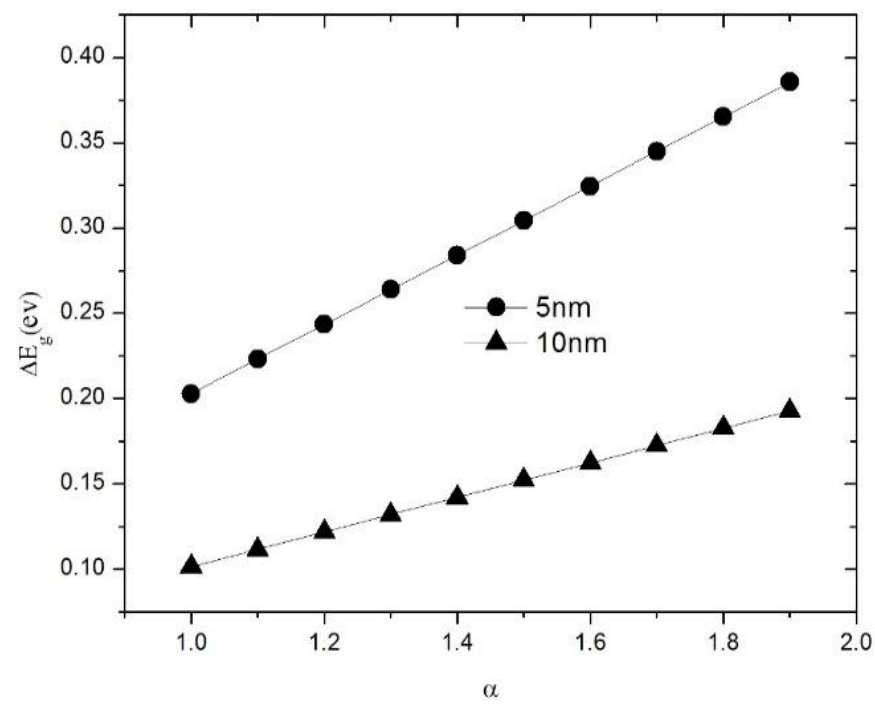

Fig.5: Deviation of size dependent band gap for Si nano particles.

The model predicts that for the given particle size, as the shape factor increases, the band gap increases. The particle shape has large effect on small particles than on large particles.

\section{CONCLUSION}

In summary, a theoretical approach has been suggested to account the influence of shape effect along with the size for static refractive index, static dielectric constant and band gap. At the nano scale range the surface to volume ratio becomes very large, so the number of atoms at the surface are very large in comparison to volume. Therefore, for nanomaterials, the number of atoms per unit volume will decrease. At the nano scale range, electron orbits contracts that causes increment in Coulomb's force which enhances restoring force. Hence the natural angular frequency of electron oscillation, which causes reduction in dielectric constant so we found that the static refractive index and static dielectric constant of $\mathrm{Si}$ and $\mathrm{Ge}$ nanoparticle, nanowire and nanofilm decrease as particle size decreases. In the present study, a simple approach is applied to compute the band gap of nano materials. It is observed that band gap increases as particle size decreases. Band gap is also temperature dependent [30]. This model is potentially applicable for those materials whose experimental data are not available.

\section{REFERENCES}

H.Lu, X.Meng, "Correlation between bandgap, dielectric constant, young modulus and melting temperature of GaN nanocrystals and their size and shape dependence”, Scientific Reports 5: 16939, 2015.

[2] M. Li, J.C. Li, Q. Jiang, "Size-Dependent Band-Gap and Dielectric Constant of Si Nanocrystals, International Journal of Modern Physics B Vol.24, Issue 15 \& 16, pp. 2297-2301, 2010.

[3] K. K. Nanda, S. N. Sahu, S. N. Behera, "Liquid drop-model for size-dependent melting of low-dimensional system", PHYSICAL REVIEW A Vol. 66, pp. 013208,2002.

[4] K. K. Nanda, "A simple classical approach for the melting temperature of inert-gas nanoparticles", Chem. Phys. Lett., Vol. 419, pp. 195-200, 2006.

[5] W.H.Qi., Physica B, "Size effect on melting temperature of nanosolids", Vol.368, pp.46-50, 2005.

[6] Q. Jiang, J. C. Li, and B. Q. Chi, "Size-dependent cohesive energy of nanocrystals", Chem. Phys. Lett., Vol. 366, pp. 551-554, 2002.

[7] W. H. Qi, M. P. Wang, and G. Y. Xu, "The particle size dependence of cohesive energy of metallic nanoparticles", Chem. Phys. Lett., Vol.372, pp. 632-634, 2003.

[8] A. D. Yoffe, "Semiconductor quantum dots and related systems: electronic, optical, luminescence and related properties of low dimensional systems", Adv. Phys. Vol.50, pp. 1-208, 2001.

[9] C. Delerue, M. Lannoo, and G. Allan, "Concept of dielectric constant for nanosized systems". Phys. Rev. B, Vol.68, pp. 115411, 2003.

[10] G. Guisbiers, Di Liu, Q. Jiang, and L. Buchaillot, "Theoretical predictions of wurtzite III-nitride nanomaterials properties". Phys. Chem. Chem. Phys., Vol.12, pp. 7203-7210, 2010.

[11] J. Zhang, "Small-scale effect on the piezoelectric potential of gallium nitride nanowires". Appl. Phys. Lett., Vol.104, pp. 253110, 2014

[12] G. Guisbiers, "Size-dependent materials properties toward a universal Equation. Nanoscale” Res. Lett., Vol.5, pp. 11321136, 2010.

[13] Feynman, Leighton, Sands, "The Feynman lectures on physics", California Institute of Technology, Vol.1, Ch.31.

[14] C kittle," Introduction to solid state physics" Wiley India Pvt. Ltd., 2015.

[15] Li. Lihong, Ma. Hansong, and Wei.Yueguang, "Sizedependent elastic modulus and vibration frequency of nanocrystals", Journal of Nanomaterials, 2011.

[16] W.H. Qi, M. P. Wang, "Size and shape dependent melting temperature of metallic nanoparticles", Materials Chemistry and Physics, Issue 16, Vol.88, pp. 280-284, 2004.

[17] W.H. Qi et al., "Shape factor for non-cylindrical nanowires", physica B, vol.403, Issue 13-16, pp. 2386-2389, 2008.

[18] G. R. Patel, N.A. Thakar, T.C. Pandya," Size and shape dependent melting temperature and thermal expansivity of metallic semiconductor nanoparticles" in the $60^{\text {th }}$ DAESSPS, AIP Conference Proceedings. 1731, 050042, 2015.

[19] N.M. Ravindra, P. Ganapathy, Jinsoo Choi, "Energy gaprefractive index relations in semiconductors" -An overview Infrared Physics \& Technology, Vol. 50, pp. 21-29, 2007

[20] R. Zallen, "The Physics of Amorphous Solids", Wily, New York, 1983.

[21] C. Delerue, M. Lannoo and G. Allan, "concept of dielectric constant for nanosized systems", Phys. Rev. B Vol.68, pp. 115411, 2003.

[22] H. G. Yoo and P. M. Fauchet, "Dielectric constant reduction in silicon nanostructure", Phys. Rev. B, Vol.77, pp. 115355, 2008. 
[23] L. Dorigoni, O. Bisi, F. Bernardini and S. Ossicini, "Electron states and luminescence transition in porous silicon", Phys Rev B, Vol. 53, pp. 4557, 1996.

[24] D. D. D. Ma, C.S.Lee, F.C.K.Au, S.Y.Tong, S.T.Lee, "Small-diameter silicon nanowire, surface Science", Vol. 299, pp.1874, 2003.

[25] X. Y. Zhao, C. M. Wei, L. Yang, and M. Y. Chou, "Quantum confinement and electronic properties of silicon nanowires" Phys. Rev. Lett., Vol.92, pp. 236805, 2004.

[26] Y. Kanemitsu, H. Uto and Y. Masumoto, "Microstructure and optical properties of free standing porous silicone films: size dependence of absorption spectra in Si nanometer sized - crystallites" Phys. Rev. B, Vol.48, pp. 2827, 1993.

[27] C. Y. Yeh, S. B. Zhang and A. Zunger, "Confinement, surface, and chemisorption effects on the optical properties of Si quantum wires", Phys Rev, Vol.40, pp. 14405, 1994.

[28] T.Van Buuren, L. N. Dinh, L. L. Chase., W. J. Siekhaus and L. J. Terminello "Changes in the electronic properties of Si Nanocrystals as a function of particle size", Phys. Rev. Lett., Vol.80, pp. 3803, 1998.

[29] P. Alfaro, A. Miranda, A.E Ramos., and M. Cruz-Irisson, "Hydrogenated Ge nanocrystals: band gap evolution with increasing size", Brazilian Journal of Physics, Vol.36, no.2A, 2006.

[30] R.K.Das "optical and electrical properties of Zno nanorods at different temperatures" International journal of scientific research in physics and applied sciences, Vol.5,Issue 6,pp.912,2017 .

\section{AUTHORS PROFILE}

Mr. G.R. Patel pursued B.Sc. and M.Sc. from North Gujarat university, Patan, Gujarat in 1993 , \& 1995. He is currently working as an assistant Professor in physics Department at Government- Science college at Gandhinagar since 2003 . He is a life member of IAPT since 2013. He has published 6 research papers in various National, International Conference Proceedings. His main research is

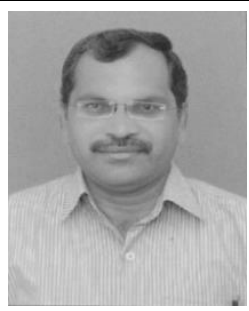
focused on size dependent properties of nanomaterials. He has 15 years of teaching experience and 03 years of research experience.

Dr. Tushar Pandya is working as an Associate Professor of Physics at St. Xavier's College, Ahmadabad. $\mathrm{He}$ obtained his Ph.D. from Gujarat University, Ahmadabad in the field of Condensed Matter Physics and has authored several research papers on this subject. He has published more than twenty-five research papers in various

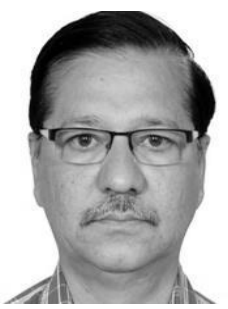

National, International journals and conference proceedings. His current research interest is in the field of High-pressure behavior of certain solids and Nano materials for the determination of the thermo physical and mechanical properties. He has 32 years of teaching experience and 17 years of research experience. 